\title{
Metric-locating-dominating sets of graphs for constructing related subsets of vertices
}

\author{
Antonio González \\ Departamento de Matemática Aplicada I, Universidad de Sevilla, Spain \\ Carmen Hernando ${ }^{1}$, Mercè Mora ${ }^{2}$ \\ Departamento de Matemáticas, Universitat Politècnica de Catalunya, Spain
}

\begin{abstract}
A dominating set $S$ of a graph is a metric-locating-dominating set if each vertex of the graph is uniquely distinguished by its distances from the elements of $S$, and the minimum cardinality of such a set is called the metric-location-domination number. In this paper, we undertake a study that, in general graphs and specific families, relates metric-locating-dominating sets to other special sets: resolving sets, dominating sets, locating-dominating sets and doubly resolving sets. We first characterize the extremal trees of the bounds that naturally involve metric-location-domination number, metric dimension and domination number. Then, we prove that there is no polynomial upper bound on the location-domination number in terms of the metric-location-domination number, thus extending a result of Henning and Oellermann. Finally, we show different methods to transform metric-locating-dominating sets into locating-dominating sets and doubly resolving sets. Our methods produce new bounds on the minimum cardinalities of all those sets, some of them concerning parameters that have not been related so far.
\end{abstract}

Keywords: metric-locating-dominating set, resolving set, dominating set, locating-dominating set, doubly resolving set

\section{Introduction}

Metric-locating-dominating sets were introduced in 2004 by Henning and Oellermann [24] combining the usefulness of resolving sets, that roughly speaking differentiate the vertices of a graph, and dominating sets, which cover the whole vertex set. Resolving sets were defined in the 1970s by Slater [43], and independently by Harary and

\footnotetext{
Email addresses: gonzalezh@us.es (Antonio González), carmen.hernando@upc.edu (Carmen Hernando), merce.mora@upc.edu (Mercè Mora)

${ }^{1}$ Partially supported by projects Gen. Cat. DGR 2017SGR1336 and MTM2015-63791-R (MINECO/FEDER)

${ }^{2}$ Partially supported by projects Gen. Cat. DGR 2017SGR1336, H2020-MSCA-RISE-2016-734922 CONNECT and MTM2015-63791-R (MINECO/FEDER)
} 
Melter [21], whereas dominating sets were introduced in the 1960s by Ore [37]. Both types of sets have received much attention in the literature because of their many and varied applications in other areas. For example, resolving sets serve as a tool for combinatorial optimization [39], game theory [20], and pharmaceutical chemistry [9]; and dominating sets are helpful to analyze computer networks [38], design codes [12], and model biological networks [23]. Although metric-locating-dominating sets are hard to handle, for entailing the complexity of the other two concepts, they have been studied in several papers, for instance [5, 26, 27], and further generalized in other works such as $[35,44]$.

Let $G=(V(G), E(G))$ be a finite, simple, undirected, and connected graph of order $n=|V(G)| \geq 2$; the distance $d(u, v)$ between two vertices $u, v \in V(G)$ is the length of a shortest $u-v$ path. We say that a subset $S \subseteq V(G)$ is a resolving set of $G$ if for every $x, y \in V(G)$ there is a vertex $u \in S$ such that $d(u, x) \neq d(u, y)$ (it is said that $S$ resolves $\{x, y\})$, and the minimum cardinality of such a set is called the metric dimension of $G$, written as $\operatorname{dim}(G)$. See [1] for a survey on this well studied graph invariant. When $S$ is also a dominating set of $G$ (i.e., every $x \in V(G) \backslash S$ has a neighbor in $S$ ), then $S$ is called a metric-locating-dominating set (MLD-set for short). The metric-locationdomination number (resp., domination number), written as $\gamma_{M}(G)$ (resp., $\gamma(G)$ ), is the minimum cardinality of an MLD-set (resp., dominating set) of $G$. Concerning specifically $\gamma(G)$, the survey [22] provides fundamental results and major research achievements in problems related to this parameter.

This paper first focuses on the intrinsic relations among MLD-sets, resolving sets and dominating sets. Indeed, the corresponding parameters for all those sets satisfy by definition

$$
\max \{\operatorname{dim}(G), \gamma(G)\} \leq \gamma_{M}(G) \leq \operatorname{dim}(G)+\gamma(G) .
$$

We consider here this chain restricted to trees; specifically, we characterize the trees for which equality occurs in (1), thereby continuing the work of Henning and Oellermann [24] that characterized the trees $T$ with $\gamma_{M}(T)=\gamma(T)$. Analogous characterizations of trees in terms of other related invariants can be found in $[3,19]$.

We also compare MLD-sets with other subsets of vertices defined by Slater [41] that are directly connected to them: the locating-dominating sets. They are dominating sets that distinguish vertices by using neighborhoods instead of distances. Locatingdominating sets are of interest for its applications; for instance, the authors of [18] have used them to approach a problem proposed by Boutin [4] that involves the metric dimension. Furthermore, locating-dominating sets have applications outside graph theory; among them: location of intruders in facilities [42], and detection of inoperable components in multiprocessor networks [5]. More formally, a locating-dominating set (LD-set for brevity) of $G$ is a dominating set $S \subseteq V(G)$ such that $N(x) \cap S \neq N(y) \cap S$ for every $x, y \in V(G) \backslash S$. The minimum cardinality of such a set, denoted by $\gamma_{L}(G)$, is the location-domination number of $G$. There is also an extensive literature on $\gamma_{L}(G)$ studying multiple aspects: complexity [13, 17], specific families [14, 16, 25, 29, 31], bounds $[2,15,22,40]$, and approximation algorithms [45]. Clearly, an LD-set is an MLD-set, and so it is also a resolving set; consequently,

$$
\operatorname{dim}(G) \leq \gamma_{M}(G) \leq \gamma_{L}(G) .
$$

See $[5,27]$ for more properties of chain (2) and bounds concerning its three parameters. 
Regarding the relation between $\gamma_{M}(G)$ and $\gamma_{L}(G)$, we propose a way to obtain LDsets from MLD-sets which helps us to extend the following result due to Henning and Oellermann.

Theorem 1.1. [24] For any tree $T$, it holds that $\gamma_{L}(T)<2 \gamma_{M}(T)$. However, there is no constant $c$ such that $\gamma_{L}(G) \leq c \gamma_{M}(G)$ for all graphs $G$.

We finally find relationships between MLD-sets and other subsets for which, so far as we are aware, no direct connection is known: the doubly resolving sets. Cáceres et al. [6] introduced doubly resolving sets as a tool for computing the metric dimension of cartesian products of graphs and, following the same spirit, Hertz [28] used them for computing the metric dimension of some hypercubes. Furthermore, different authors have provided other interesting applications of them; for instance, in source location. Indeed, Chen and Wang [11] utilized doubly resolving sets for modeling the problem of locating the source of a diffusion in a complex network, which is necessary for controlling and preventing epidemic risks. See $[8,10]$ for a similar approach and [30] for more information on general source location. Doubly resolving sets, that somehow distinguish vertices in two ways by means of distances, are formally defined as follows. Two vertices $u, v \in V(G)$ doubly resolve a pair $\{x, y\} \subseteq V(G)$ if $d(u, x)-d(u, y) \neq d(v, x)-d(v, y)$. A set $S \subseteq V(G)$ is a doubly resolving set of $G$ if every pair $\{x, y\} \subseteq V(G)$ is doubly resolved by two vertices of $S$ (it is said that $S$ doubly resolves $\{x, y\}$ ), and the minimum cardinality of such a set is denoted by $\psi(G)$. Thus, a doubly resolving set is also a resolving set, and so

$$
\operatorname{dim}(G) \leq \psi(G) .
$$

Although it is not straightforward to deduce any relation between $\psi(G)$ and $\gamma_{M}(G)$ from their definitions, we provide here bounds on $\psi(G)$ in terms of $\gamma_{M}(G)$ by generating doubly resolving sets from MLD-sets. We thus obtain, for specific classes and general graphs, similar chains to expression (2) that include $\psi(G)$. For more references on $\psi(G)$ containing algorithmic studies and relations with other graph parameters, we refer the reader to $[7,33,34]$ for results on specific families of graphs and $[10,32,36]$ for results on general graphs.

The paper is organized as follows. In Section 2, we characterize all trees achieving the extremal values in expression (1). We then show in Sections 3 and 4 how to construct LD-sets and doubly resolving sets from MLD-sets in arbitrary graphs and specific families, thus producing bounds on the corresponding parameters. Specifically, we prove in Section 3 that $\gamma_{L}(G) \leq \gamma_{M}^{2}(G)$ whenever $G$ has no cycles of length 4 or 6 but, for arbitrary graphs, any upper bound on $\gamma_{L}(G)$ in terms of $\gamma_{M}(G)$ has at least exponential growth; in Section 4, we provide the bounds $\psi(G) \leq \gamma_{M}(G)$ for graphs $G$ with girth at least 5 , and $\psi(G) \leq \gamma_{M}(G)+\gamma(G)$ for any graph $G$. We conclude the paper with some remarks and open problems in Section 5 .

\section{MLD-sets of trees}

Henning and Oellermann [24] provided a formula for the metric-location-domination number of trees and characterized the trees $T$ with $\gamma_{M}(T)=\gamma(T)$, giving both results in terms of support vertices (see Theorem 2.1 below). Recall that a vertex $u$ of a tree $T$ is a support vertex whenever it is adjacent to some leaf (i.e., a vertex of degree 1), and 
it is a strong support vertex if there are two or more leaves adjacent to $u$. We denote by $\mathcal{S}(T)$ (resp., $\mathcal{S}^{\prime}(T)$ ) the set of support (resp., strong support) vertices of $T ; \ell^{\prime}(T)$ is the number of leaves adjacent to a strong support vertex.

Theorem 2.1. [24] For any tree T, the following statements hold:

(i) $\gamma_{M}(T)=\gamma(T)+\ell^{\prime}(T)-\left|\mathcal{S}^{\prime}(T)\right|$.

(ii) $\gamma_{M}(T)=\gamma(T)$ if and only if $\mathcal{S}^{\prime}(T)=\emptyset$.

As the authors observed, any MLD-set must contain, for each support vertex $u$, either all the leaves adjacent to $u$ or all but one of the leaves adjacent to $u$ as well as vertex $u$. This observation leads us to see that $\ell(T) \leq \gamma_{M}(T)$, where $\ell(T)$ denotes the total number of leaves of any tree $T$. Hence, $\operatorname{since} \operatorname{dim}(T)<\ell(T)$ (see [9]), expression (1) now becomes

$$
\max \{\ell(T), \gamma(T)\} \leq \gamma_{M}(T) \leq \operatorname{dim}(T)+\gamma(T) .
$$

This section follows the same spirit as Henning and Oellermann [24], who characterized in statement (ii) of Theorem 2.1 the extremal trees for expression (4) with $\gamma_{M}(T)=$ $\gamma(T)$. Indeed, we characterize the remaining extremal cases: $\gamma_{M}(T)=\operatorname{dim}(T)+\gamma(T)$ in Theorem 2.2, and $\gamma_{M}(T)=\ell(T)$ in Theorem 2.3. To do this, we first recall the following terminology extracted from [9]. A vertex $u \in V(T)$ of degree at least 3 is called a major vertex of $T$, and a leaf $x \in V(T)$ is a terminal vertex of $u$ if the major vertex closest to $x$ is $u$. The terminal degree of a major vertex $u$, written as ter $(u)$, is the number of its terminal vertices, and $u$ is an exterior major vertex of $T$ if it has positive terminal degree.

Theorem 2.2. Let $T$ be a tree different from a path. Then, the following statements are equivalent:

(i) $\gamma_{M}(T)=\operatorname{dim}(T)+\gamma(T)$.

(ii) $\operatorname{dim}(T)=\ell^{\prime}(T)-\left|\mathcal{S}^{\prime}(T)\right|$.

(iii) Every exterior major vertex $u$ with $\operatorname{ter}(u) \geq 2$ is the support vertex of all its terminal vertices.

(iv) Any path joining two leaves of $T$ at distance greater than 2 contains at least two major vertices.

Proof. $(i \Longleftrightarrow i i)$ This equivalence is guaranteed by statement (i) of Theorem 2.1.

(ii $\Longleftrightarrow$ iii) It is known that any set $S \subseteq V(T)$ composed by all but one of the terminal vertices of each exterior major vertex $u$ is a minimum resolving set of $T$ (see Theorem 5 of [9] and its proof). Thus, let $S \subseteq V(T)$ be such a set, and note that, as any strong support vertex is an exterior major vertex, then $S$ must contain all but one of the leaves adjacent to $u$; consequently, $\operatorname{dim}(T) \geq \ell^{\prime}(T)-\left|\mathcal{S}^{\prime}(T)\right|$. Hence, if $\operatorname{dim}(T)=\ell^{\prime}(T)-\left|\mathcal{S}^{\prime}(T)\right|$ then $S$ is formed only by all but one of the leaves adjacent to each strong support vertex.

Let $u$ be an exterior major vertex with $\operatorname{ter}(u) \geq 2$, and let $x$ and $y$ be two terminal vertices of $u$. On the contrary, let us assume for instance that $x \notin N(u)$, and let $v$ be the only neighbor of $u$ in the $u-x$ path. Clearly, $u$ is the support vertex of $y$ because otherwise there is $v^{\prime} \in N(u) \backslash\{y\}$ in the $u-y$ path but $\left\{v, v^{\prime}\right\}$ would not be resolved by $S$ since no vertex in either the $u-x$ path or the $u-y$ path is in $S$ (by construction of set $S)$. Furthermore, one of the leaves adjacent to $u$, say $z$, is not in $S$ but reasoning as 
before yields that $S$ does not resolve $\{v, z\}$; a contradiction since $S$ is a resolving set of $T$. Therefore, $u$ is the support vertex of each of its terminal vertices.

Reciprocally, let us suppose that every exterior major vertex $u$ with $\operatorname{ter}(u) \geq 2$ is the support vertex of its terminal vertices. In such a case $u \in \mathcal{S}^{\prime}(T)$. Thus, any set $S \subseteq V(T)$ composed by all but one of the leaves adjacent to each strong support vertex is a resolving set, since it contains all but one of the terminal vertices of each exterior major vertex. Moreover, since $S$ is a resolving set satisfying $|S|=\ell^{\prime}(T)-\left|\mathcal{S}^{\prime}(T)\right|$ and $\operatorname{dim}(T) \geq \ell^{\prime}(T)-\left|\mathcal{S}^{\prime}(T)\right|$, as we have seen at the beginning of this proof, we conclude that $S$ is a minimum resolving set and $\operatorname{dim}(T)=\ell^{\prime}(S)-\left|\mathcal{S}^{\prime}(T)\right|$.

(iii $\Longleftrightarrow i v$ ) Let us suppose that every exterior major vertex $u$ with $\operatorname{ter}(u) \geq 2$ is adjacent to each of its terminal vertices. Given two leaves $x$ and $y$ at distance greater than 2 , there is at least one major vertex $u$ in the $x-y$ path since $G$ is not isomorphic to a path. Furthermore, this vertex $u$ cannot be unique since otherwise $x$ and $y$ are terminal vertices of $u$ with either $d(u, x) \geq 2$ or $d\left(u^{\prime}, y\right) \geq 2$, that is, $u$ is not the support vertex of either $x$ or $y$, which is impossible. Reciprocally, let us assume statement (iv) to hold true, and let $u$ be an exterior major vertex with $\operatorname{ter}(u) \geq 2$. It is easily seen that no terminal vertex $x$ of $u$ verifies $d(u, x) \geq 2$ since otherwise $d(x, y) \geq 3$ for any other terminal vertex $y$ of $u$, setting $u$ as the only major vertex in the $x-y$ path, which contradicts statement (iv).

We want to remark that, although paths are not considered in the preceding result, it is easy to check that $P_{3}$ is the only path satisfying $\gamma_{M}\left(P_{n}\right)=\gamma\left(P_{n}\right)+\operatorname{dim}\left(P_{n}\right)$ since $\gamma_{M}\left(P_{n}\right)=\gamma\left(P_{n}\right)$ whenever $n \neq 3$ (by statement (i) of Theorem 2.1), and $\operatorname{dim}\left(P_{n}\right)=1$ for all $n \geq 1$ (see for instance [9]).

Theorem 2.3. Let $T$ be a tree different from the path $P_{2}$. Then, the following statements are equivalent:

(i) $\gamma_{M}(T)=\ell(T)$.

(ii) $\gamma(T)=|\mathcal{S}(T)|$.

(iii) For every $u \in V(T)$, there exists a leaf at distance at most 2 from $u$.

Proof. $(i \Longleftrightarrow i i)$ By statement (i) of Theorem 2.1, $\gamma_{M}(T)=\ell(T)$ is equivalent to $\gamma(T)=$ $\ell(T)-\ell^{\prime}(T)+\left|\mathcal{S}^{\prime}(T)\right|$ but $\ell(T)-\ell^{\prime}(T)$ is the number of non-strong support vertices, i.e. $|\mathcal{S}(T)|-\left|\mathcal{S}^{\prime}(T)\right|$, which gives $\gamma(T)=|\mathcal{S}(T)|$.

(ii $\Longleftrightarrow$ iii) Let $S \subseteq V(T)$ be a minimum dominating set of $T$. Observe that, when replacing any leaf of $S$ by its corresponding support vertex, the resulting set is still a dominating set without leaves and containing each support vertex of $T$ (since all leaves must be dominated by vertices of $S$ ). Thus, we may assume $\mathcal{S}(T) \subseteq S$. Since $S$ has minimum cardinality, it follows that $\gamma(T) \geq|\mathcal{S}(T)|$. Therefore, $\gamma(T)=|\mathcal{S}(T)|$ if and only if $\mathcal{S}(T)$ is a minimum dominating set of $T$, i.e., every $u \in V(T)$ is either in $\mathcal{S}(T)$ or has a neighbor in $\mathcal{S}(T)$. Equivalently, $\gamma(T)=|\mathcal{S}(T)|$ if and only if every vertex $u$ is either a support vertex $(d(u, x)=1$ for some leaf $x \in N(u))$, or $u$ is a leaf $(d(u, u)=0)$, or $u$ is adjacent to a support vertex, say $v(d(u, x)=2$ for some leaf $x \in N(v))$.

\section{MLD-sets versus LD-sets}

In view of the relationship $\gamma_{M}(G) \leq \gamma_{L}(G)$ given in expression (2), it is natural to look for upper bounds on $\gamma_{L}(G)$ in terms of $\gamma_{M}(G)$ as Henning and Oellermann [24] did 
in Theorem 1.1. In this section, we extend this result by first providing a wide class of graphs $G$ whose MLD-sets can be extended to LD-sets; this leads us to the upper bound $\gamma_{L}(G) \leq \gamma_{M}^{2}(G)$. We also prove that $\gamma_{L}(G)$ is at least exponential in $\gamma_{M}(G)$ for arbitrary graphs $G$.

Let $G$ be a graph not having the cycles $C_{4}$ or $C_{6}$ as a subgraph, and let $S$ be any subset of $V(G)$. We assign to every pair $u, v \in S$ a set of vertices $\pi(u, v)$ given by $\left\{u^{\prime}, v^{\prime}\right\}$ whenever there exists a $u-v$ path $\left(u, u^{\prime}, v^{\prime}, v\right)$ (that is unique because of the $C_{4^{-}}$and $C_{6}$-free condition), and $\emptyset$ otherwise. Let $\pi(S)=\bigcup_{u, v \in S} \pi(u, v)$.

Proposition 3.1. Let $G$ be a graph not containing $C_{4}$ or $C_{6}$ as a subgraph. For every $M L D$-set $S \subseteq V(G)$, the set $S \cup \pi(S)$ is an LD-set of $G$. Consequently, $\gamma_{L}(G) \leq \gamma_{M}^{2}(G)$.

Proof. Let $\bar{S}=S \cup \pi(S)$. Since $S \subseteq \bar{S}$ is a dominating set of $G$, we only need to check that $N(x) \cap \bar{S} \neq N(y) \cap \bar{S}$ for every $x, y \in V(G) \backslash \bar{S}$. On the contrary, let us assume the existence of two vertices $x, y \in V(G) \backslash \bar{S}$ so that $N(x) \cap \bar{S}=N(y) \cap \bar{S}$. Let $u$ and $v$ be vertices in $S$ such that $x \in N(u)$ and $y \in N(v)$. If $u \neq v$ then either $x \notin N(v)$ or $y \notin N(u)$ (otherwise $(u, x, v, y)$ would be a cycle on 4 vertices of $G$, which leads to a contradiction), and so $N(x) \cap \bar{S} \neq N(y) \cap \bar{S}$. Hence, $u=v$. Moreover, since the existence of a vertex $w \in N(x) \cap N(y)$ different from $u$ produces the cycle $(u, x, w, y)$, which cannot exist, then we have that $N(x) \cap N(y)=\{u\}$, and so $N(x) \cap S=N(y) \cap S=\{u\}$.

Let $z \in V(G) \backslash S$ be a neighbor of either $x$ or $y$ (that exists because otherwise $N(x)=N(y)=\{u\}$ and so the pair $\{x, y\}$ is not resolved by $S$, which is impossible since $S$ is a resolving set). Assuming without loss of generality $z \in N(x)$, we have that $z \notin N(y)$ since we have seen that $N(x) \cap N(y)=\{u\}$. On the other hand, there is a vertex $u^{\prime} \in S$ dominating $z$. If $u^{\prime} \neq u$ then $\pi\left(u, u^{\prime}\right)=\{x, z\} \subseteq \pi(S)$; a contradiction since $x \notin \pi(S)$. Therefore, $u=u^{\prime}$ and $N(z) \cap S=\{u\}$.

Let $z^{\prime} \in V(G) \backslash S$ be such that either $z^{\prime} \in N(x) \backslash N(z)$ or $z^{\prime} \in N(z) \backslash N(x)$ (which exists since otherwise $N[x]=N[z]=\{x, z, u\}$ and so the pair $\{x, z\}$ is not resolved by $S$ ). Also, let $u^{\prime \prime} \in S$ dominating $z^{\prime}$, which must be different from $u$ since otherwise we could obtain the cycle $\left(u, z^{\prime}, x, z\right)$ when $z^{\prime} \in N(x) \backslash N(z)$, or the cycle $\left(u, x, z, z^{\prime}\right)$ when $z^{\prime} \in N(z) \backslash N(x)$. If $z^{\prime} \in N(x) \backslash N(z)$ then $\pi\left(u, u^{\prime \prime}\right)=\left\{x, z^{\prime}\right\} \subseteq \pi(S)$, which contradicts $x \notin \bar{S}=S \cup \pi(S)$. Hence, $z^{\prime} \in N(z) \backslash N(x)$ and so $\pi\left(u, u^{\prime \prime}\right)=\left\{z, z^{\prime}\right\} \subseteq \pi(S)$. Consequently, $z \in \bar{S}$ but $z \in N(x)$ and $z \notin N(y)$, which implies that $N(x) \cap \bar{S} \neq N(y) \cap \bar{S}$ thus producing a contradiction.

We have thus proved that $\bar{S}$ is an LD-set of $G$. To complete the proof, observe that, for any pair $u, v \in S$, the set $\pi(u, v)$ may intersect either $S$ or $\pi\left(u^{\prime}, v^{\prime}\right)$ for another pair $u^{\prime}, v^{\prime} \in$ $S$. Consequently, $|\pi(S)| \leq 2\left(\begin{array}{c}(S \mid) \\ 2\end{array}\right)$ and so $|\bar{S}| \leq|S|+|\pi(S)| \leq|S|^{2}$. Therefore, choosing $S$ with minimum cardinality yields $\gamma_{L}(G) \leq|\bar{S}| \leq|S|^{2}=\gamma_{M}^{2}(G)$, as required.

Henning and Oellermann [24] showed that there is no linear upper bound on $\gamma_{L}(G)$ in terms of $\gamma_{M}(G)$ by building up a suitable family of graphs $G$ with $\gamma_{L}(G)>c \gamma_{M}(G)$ for any constant $c$. However, the graphs of that family satisfy $\gamma_{L}(G)<\gamma_{M}^{2}(G)$. A natural question that arises at this point is to determine if the parameter $\gamma_{L}(G)$ is polynomial in $\gamma_{M}(G)$. Nevertheless, this is far from being true as we next prove; concretely, we provide a family of graphs $G$ such that $\gamma_{L}(G)$ is at least exponential in $\gamma_{M}(G)$.

Theorem 3.2. There is no polynomial function $f$ such that $\gamma_{L}(G) \leq f\left(\gamma_{M}(G)\right)$ for all graphs $G$. 
Proof. To prove this result, we construct a family of graphs $G_{s}$ such that $\gamma_{L}\left(G_{s}\right) \geq$ $2^{\gamma_{M}\left(G_{s}\right)-2}-1$ as follows. For a positive integer $s$, let $A=\left\{a_{i}: i=0, \ldots, 2^{s+1}-1\right\}$, $B=\left\{b_{i}: i=0, \ldots, 2^{s+1}-1\right\}$ and $C=\left\{c_{i}: i=0, \ldots, s\right\}$. The graph $G_{s}$ has vertex set $V\left(G_{s}\right)=\{p\} \cup A \cup B \cup C$ and edge set given by the pairs $p a_{i}$ and $a_{i} b_{i}$ for every $i \in\left\{0, \ldots, 2^{s+1}-1\right\}$, and $b_{i} c_{j}$ whenever the binary representation of $i$ has a 1 in its $j$-th position (Figure 1 illustrates the case for $s=2$ ).

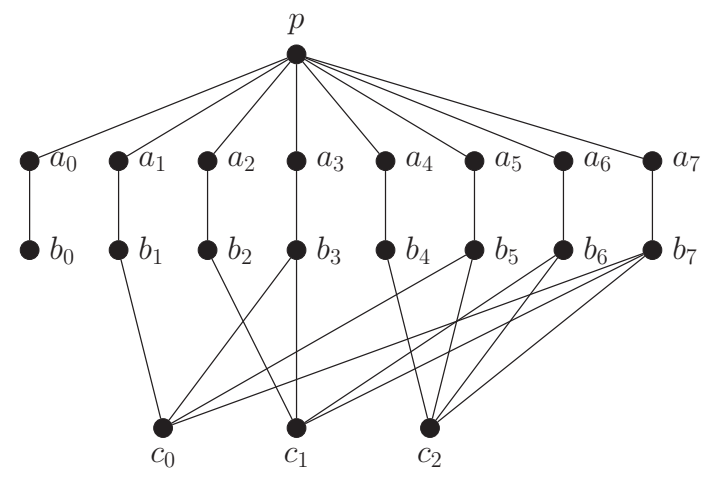

Figure 1: The graph $G_{2}$.

It is easy to check that the set $\{C\} \cup\{p\} \cup\left\{b_{0}\right\}$ is an MLD-set of $G_{s}$, which implies that

$$
\gamma_{M}\left(G_{s}\right) \leq s+3
$$

On the other hand, each LD-set $S$ of $G_{s}$ must contain either vertex $a_{i}$ or vertex $b_{i}$ for all but at most one $i \in\left\{0, \ldots, 2^{s+1}-1\right\}$. Indeed, if there exist $i$ and $j$ such that $a_{i}, b_{i}, a_{j}, b_{j} \notin S$ then

$$
N\left(a_{i}\right) \cap S=N\left(a_{j}\right) \cap S=\left\{\begin{array}{lcc}
\{p\} & \text { if } & p \in S \\
\emptyset & & \text { otherwise }
\end{array}\right.
$$

But this is impossible since $S$ is an LD-set of $G$. Thus,

$$
\gamma_{L}\left(G_{s}\right) \geq 2^{s+1}-1 \text {. }
$$

Therefore, combining inequalities (5) and (6) yields $\gamma_{L}\left(G_{s}\right) \geq 2^{\gamma_{M}\left(G_{s}\right)-2}-1$, which gives the result.

\section{Doubly resolving sets from MLD-sets}

In this section, we show how useful MLD-sets can be for constructing doubly resolving sets of graphs. Indeed, given an MLD-set of a graph $G$ with girth at least 5 we construct a doubly resolving set of the same size (recall that the girth, $\mathrm{g}(G)$, of $G$ is the length of a shortest cycle of $G$ ); when $G$ is any graph, our method also implies the use of dominating sets. In both cases we obtain bounds that involve $\psi(G)$ and $\gamma_{M}(G)$ (Proposition 4.4 and 
Theorem 4.6), giving rise to chains similar to expression (2) but including the invariant $\psi(G)$ (Corollaries 4.5 and 4.7). We start with the following two lemmas that are the key to relate MLD-sets to doubly resolving sets.

Lemma 4.1. Let $G$ be a graph and let $S$ be an $M L D$-set of $G$. Then, every pair $x, y \in$ $V(G) \backslash S$ is doubly resolved by $S$.

Proof. Given any two vertices $x, y \in V(G) \backslash S$, we shall prove that there exist $u, v \in S$ such that $d(u, x)-d(u, y) \neq d(v, x)-d(v, y)$. Indeed, let $u^{\prime}, v^{\prime} \in S$ such that $x \in N\left(u^{\prime}\right)$ and $y \in N\left(v^{\prime}\right)$, which exist since $S$ is a dominating set of $G$. We distinguish two cases:

1. $u^{\prime}=v^{\prime}$. We have $d\left(u^{\prime}, x\right)-d\left(u^{\prime}, y\right)=1-1=0$ and, since $S$ is a resolving set, there is a vertex $w \in S \backslash\left\{u^{\prime}\right\}$ such that $d(w, x) \neq d(w, y)$, which implies that $d(w, x)-d(w, y) \neq 0$. Therefore, we set $\{u, v\}=\left\{u^{\prime}, w\right\}$.

2. $u^{\prime} \neq v^{\prime}$. We can assume that $x \notin N\left(v^{\prime}\right)$ and $y \notin N\left(u^{\prime}\right)$ (otherwise we proceed as in the previous case) and so $d\left(u^{\prime}, y\right), d\left(v^{\prime}, x\right)>1$. Hence, $d\left(u^{\prime}, x\right)-d\left(u^{\prime}, y\right)<0$ and $d\left(v^{\prime}, x\right)-d\left(v^{\prime}, y\right)>0$, so we can take $\{u, v\}=\left\{u^{\prime}, v^{\prime}\right\}$.

Lemma 4.2. Let $S$ be an $M L D$-set of a graph $G$, and let $u \in S$ and $x \in V(G) \backslash S$ such that $\{u, x\}$ is not doubly resolved by $S$. Then, $N(x) \cap S=\{u\}$. Furthermore, $x$ is the only vertex of $V(G) \backslash S$ such that $\{u, x\}$ is not doubly resolved by $S$.

Proof. First, we prove that $N(x) \cap S=\{u\}$. Observe that, for every $v \in S$,

$$
d(u, u)-d(u, x)=d(v, u)-d(v, x)
$$

since pair $\{u, x\}$ is not doubly resolved by $S$ (in particular by $u$ and $v$ ). As $S$ is a dominating set, there is some vertex $v^{*} \in S$ with $x \in N\left(v^{*}\right)$, and so setting $v=v^{*}$ in (7) yields $-d(u, x)=d\left(v^{*}, u\right)-1$. Necessarily, $d\left(v^{*}, u\right)=0$ and $d(u, x)=1$, which implies that $v^{*}=u$ and (7) becomes

$$
d(v, x)=d(v, u)+1
$$

for each $v \in S$. Hence, $d(v, x)>1$ whenever $v \in S \backslash\{u\}$, and so $N(x) \cap S=\{u\}$.

Now, we show that there is no other vertex $x^{\prime} \in V(G) \backslash S$ different from $x$ so that $\left\{u, x^{\prime}\right\}$ is not doubly resolved by $S$. Let us assume on the contrary the existence of such a vertex $x^{\prime}$. Reasoning as above, we easily get $d\left(v, x^{\prime}\right)=d(v, u)+1$ for any $v \in S$, which combined with (8) gives $d(v, x)=d\left(v, x^{\prime}\right)$; a contradiction since $S$ is a resolving set of $G$.

Observation 4.3. For any subset of vertices $S$ of a graph $G$, it is obvious that any pair $\{u, v\} \subseteq S$ is doubly resolved by $u$ and $v$.

Regarding Lemmas 4.1 and 4.2 and Observation 4.3, it is natural to ask whether MLD-sets $S$ doubly resolve pairs $\{u, x\}$ with $u \in S$ and $x \in V(G) \backslash S$, thus implying that MLD-sets would be doubly resolving sets (and so $\psi(G) \leq \gamma(G)$ ). Unfortunately, this is not true in general as graph $H_{t}$ depicted in Figure 2 shows, because the set $\left\{a_{1}, \ldots, a_{t}\right\}$ is an MLD-set of $H_{t}$ but it does not doubly resolve any pair $\left\{a_{i}, c_{i}\right\}$. Also, $\psi\left(H_{t}\right)=2 t=2 \gamma_{M}(G)$ (since all doubly resolving sets satisfy $\left|\left\{a_{i}, b_{i}, c_{i}\right\} \cap S\right| \geq 2$ for each $i$ ) and so the bound $\psi(G) \leq \gamma(G)$ does not hold for all graphs $G$. Furthermore, 
even adding the extra condition $\mathrm{g}(G) \geq 5$, MLD-sets are not necessarily doubly resolving sets (see the graph $H_{t}^{\prime}$ of Figure 3 ). However, for this class of graphs, we next describe how to modify the elements of any MLD-set to obtain a doubly resolving set of the same cardinality, thereby producing the bound $\psi(G) \leq \gamma_{M}(G)$.

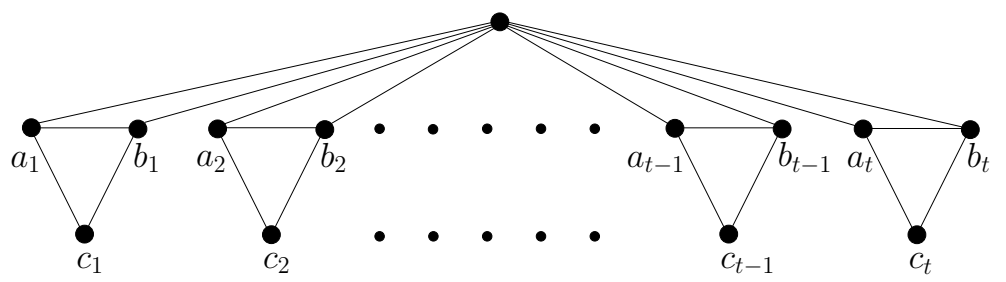

Figure 2: The graph $H_{t}$, with $t \geq 2$, for which $\left\{a_{1}, \ldots, a_{t}\right\}$ and $\left\{b_{1}, \ldots, b_{t}, c_{1}, \ldots, c_{t}\right\}$ are, respectively, a minimum MLD-set and a minimum doubly resolving set.

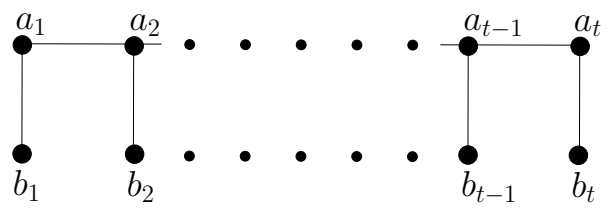

Figure 3: The graph $H_{t}^{\prime}$, with $t \geq 1$, where $\left\{a_{1}, \ldots, a_{t}\right\}$ is a minimum MLD-set but it does not doubly resolve any pair $\left\{a_{i}, b_{i}\right\}$.

Let $S$ be an MLD-set of a graph $G$ with $g(G) \geq 5$. Observe that every $u \in S$ has at most one neighbor of degree 1 in $V(G) \backslash S$ (otherwise $S$ would not be a resolving set). Let $\widetilde{u}$ be such a neighbor if it exists, and $u$ otherwise. Note that, by construction, $\widetilde{u} \neq \widetilde{v}$ for any two different vertices $u, v \in S$. The following result proves that replacing each $u \in S$ with $\widetilde{u}$ yields a doubly resolving set of $G$ whenever $g(G) \geq 5$.

Proposition 4.4. Let $G$ be a graph with girth $\mathrm{g}(G) \geq 5$ and $G \neq P_{2}$. For any $M L D$-set $S \subseteq V(G)$, the set $\widetilde{S}=\{\widetilde{u}: u \in S\}$ is a doubly resolving set of $G$. Consequently,

$$
\psi(G) \leq \gamma_{M}(G)
$$

and this bound is tight.

Proof. We begin by noticing that, given a vertex $u \in S$, we have that

$$
d(\widetilde{u}, x)-d(\widetilde{u}, y)=d(u, x)-d(u, y)
$$

for any two vertices $x, y \neq \widetilde{u}$ (because if $\widetilde{u} \neq u$ then $N(\widetilde{u})=\{u\}$, and so $d(\widetilde{u}, x)-d(\widetilde{u}, y)=$ $(d(u, x)+1)-(d(u, y)+1))$. To prove that $\widetilde{S}$ is a doubly resolving set, we first show that $\widetilde{S}$ doubly resolves at least the same pairs as $S$. Indeed, let $\{x, y\} \subseteq V(G)$ be a pair that is doubly resolved by $S$, i.e., there exist $u, v \in S$ such that $d(u, x)-d(u, y) \neq d(v, x)-d(v, y)$. We shall see that $\widetilde{u}$ and $\widetilde{v}$ (which must be different) doubly resolve $\{x, y\}$. Clearly, if 
$x, y \notin\{\widetilde{u}, \widetilde{v}\}$ then $\widetilde{u}, \widetilde{v}$ doubly resolve $\{x, y\}$ by $(9)$; and if $\{x, y\}=\{\widetilde{u}, \widetilde{v}\}$ then $\widetilde{u}, \widetilde{v}$ also doubly resolve $\{x, y\}$ by Observation 4.3. So, it only remains to prove that $\widetilde{u}, \widetilde{v}$ doubly resolve $\{x, y\}$ whenever $|\{\widetilde{u}, \widetilde{v}\} \cap\{x, y\}|=1$. Thus, let us assume without loss of generality that $x=\widetilde{u}$ and $y \neq \widetilde{v}$. We distinguish two cases.

Case 1. $u=\widetilde{u}$ : We have $d(\widetilde{u}, x)-d(\widetilde{u}, y)=d(u, x)-d(u, y)$ and, by $(9), d(\widetilde{v}, x)-$ $d(\widetilde{v}, y)=d(v, x)-d(v, y)$. But $d(u, x)-d(u, y) \neq d(v, x)-d(v, y)$ by assumption, which implies that $\widetilde{u}$ and $\widetilde{v}$ doubly resolves $\{x, y\}$.

Case 2. $u \neq \widetilde{u}$ : By the triangle inequality, $d(\widetilde{v}, y) \leq d(\widetilde{v}, u)+d(u, y) \leq(d(\widetilde{v}, \widetilde{u})-$ $1)+(d(\widetilde{u}, y)-1)$, or equivalently $-d(\widetilde{u}, y) \leq d(\widetilde{v}, x)-d(\widetilde{v}, y)-2$ since $\widetilde{u}=x$. Thus, $d(\widetilde{u}, x)-d(\widetilde{u}, y) \leq d(\widetilde{v}, x)-d(\widetilde{v}, y)-2<d(\widetilde{v}, x)-d(\widetilde{v}, y)$, and then $\{x, y\}$ is doubly resolved by $\widetilde{u}$ and $\widetilde{v}$.

Now, we show that $\widetilde{S}$ also doubly resolves the pairs $\{x, y\}$ not being doubly resolved by $S$. By Lemmas 4.1 and 4.2 and Observation 4.3, we can assume that $x \in S, y \in V(G) \backslash S$ and $N(y) \cap S=\{x\}$, being $y$ the only vertex in $V(G) \backslash S$ so that $\{x, y\}$ is not doubly resolved by $S$. Furthermore, we can prove that $N(y)=\{x\}$ (this is shown below), which implies that $\widetilde{x}=y \in \widetilde{S}$. Thus, pair $\{x, y\}$ is doubly resolved by $y \in \widetilde{S}$ and any vertex $v \in \widetilde{S} \backslash\{y\}$ since $d(y, x)-d(y, y)=1 \neq-1=d(v, x)-d(v, y)$ (note that such a vertex $v$ exists since $|S| \geq \gamma_{M}(G) \geq 2$ as $G ¥ P_{2}$; see for instance [5]). Therefore, we have proved that $\widetilde{S}$ is a doubly resolving set of $G$ of cardinality $|S|$, which gives $\psi(G) \leq \gamma_{M}(G)$ by choosing $S$ of minimum cardinality. Moreover, this bound is tight because the graph $H_{t}^{\prime}$ of Figure 3 satisfies $\psi\left(H_{t}^{\prime}\right)=\gamma_{M}\left(H_{t}^{\prime}\right)=t$ (it is easy to see that $\left\{b_{1}, \ldots, b_{t}\right\}$ is the unique minimum doubly resolving set of $H_{t}^{\prime}$; see [6] for details).

To finish the proof, it only remains to check that any pair $\{x, y\}$ that is not doubly resolved by $S$ satisfies $N(y)=\{x\}$. On the contrary, let us suppose the existence of a vertex $z \in N(y) \backslash\{x\}$, which is not in $S$ since $N(y) \cap S=\{x\}$. As $S$ is an MLD-set, there is a vertex $w \in S \cap N(z)$, which must be different from $x$ since $g(G) \geq 5$. For the same reason, we have that $d(w, x) \geq 2$, and also that $d(w, y)=2$. Thus, $d(w, x)-d(w, y) \geq 0$ but $d(x, x)-d(x, y)=-1$, so $w, x \in S$ doubly resolve $\{x, y\}$; a contradiction.

This last result, together with expression (3), allows us to place $\psi(G)$ into the chain of expression (2) as the following corollary shows.

Corollary 4.5. Let $G$ be a graph with girth $g(G) \geq 5$. Then,

$$
\operatorname{dim}(G) \leq \psi(G) \leq \gamma_{M}(G) \leq \gamma_{L}(G) .
$$

Now, we provide a bound on $\psi(G)$ for arbitrary graphs $G$. To do this, we proceed similarly as in the proof of Proposition 4.4 but using also dominating sets.

Theorem 4.6. For every graph $G$, it holds that

$$
\psi(G) \leq \gamma_{M}(G)+\gamma(G)
$$

and this bound is tight.

Proof. Let $S_{1}$ and $S_{2}$ be respectively a minimum MLD-set and a minimum dominating set of $G$. Also, let $\{x, y\} \subseteq V(G)$ be a pair that is not doubly resolved by $S=S_{1} \cup S_{2}$. Since set $S$ is in particular an MLD-set, by Lemmas 4.1 and 4.2 and Observation 4.3, we can assume $x \in S, y$ to be the only vertex of $V(G) \backslash S$ so that $\{x, y\}$ is not doubly resolved 
by $S$, and $N(y) \cap S=\{x\}$. Furthermore, $x \in S_{1} \cap S_{2}$ because $x \in S_{1} \backslash S_{2}$ (analogous for $x \in S_{2} \backslash S_{1}$ ) implies that there is $u \in S_{2}$ dominating $y$ since $S_{2}$ is a dominating set, which contradicts $N(y) \cap S=\{x\}$.

Let $S^{\prime}$ be the set of vertices $y \in V(G) \backslash S$ so that $\{x, y\}$ is not doubly resolved by $S$ for some $x \in S_{1} \cap S_{2}$ (note that $\left|S^{\prime}\right| \leq\left|S_{1} \cap S_{2}\right|$ by the uniqueness of each vertex $y \in V(G) \backslash S$ ). Clearly, $S \cup S^{\prime}$ is a doubly resolving set of $G$ and has cardinality $|S|+\left|S^{\prime}\right| \leq\left|S_{1} \cup S_{2}\right|+\left|S_{1} \cap S_{2}\right|=\left|S_{1}\right|+\left|S_{2}\right|=\gamma_{M}(G)+\gamma(G)$, which yields the expected bound. To prove tightness, we consider the graph $H_{t}$ of Figure 2 which verifies $\psi\left(H_{t}\right)=2 t$ and $\gamma_{M}\left(H_{t}\right)=\gamma\left(H_{t}\right)=t$ for each $t \geq 2$.

Combining Theorem 4.6 and the fact that $\gamma(G) \leq \gamma_{M}(G)$ for any graph $G$, we achieve the following chain that is similar to expression (2) and includes $\psi(G)$.

Corollary 4.7. For every graph $G$, it holds that

$$
\operatorname{dim}(G) \leq \psi(G) \leq 2 \gamma_{M}(G) \leq 2 \gamma_{L}(G) .
$$

We remark that tightness in the bound $\psi(G) \leq 2 \gamma_{M}(G)$ is guaranteed by the graph $H_{t}$ of Figure 2.

Finally, we propose the following conjecture that is supported by Proposition 4.4 since $\gamma_{M}(G) \leq \operatorname{dim}(G)+\gamma(G)$.

Conjecture 4.8. For every graph $G$, it holds that

$$
\psi(G) \leq \operatorname{dim}(G)+\gamma(G) .
$$

\section{Concluding remarks and open questions}

In this paper, we have first characterized the trees $T$ in the cases $\gamma_{M}(T)=\operatorname{dim}(T)+$ $\gamma(T)$ and $\gamma_{M}(T)=\ell(T)$, thus completing the study initiated by Henning and Oellermann in [24]. We have then introduced new techniques that use MLD-sets to construct LD-sets and doubly resolving sets, which have important applications in many areas as we have pointed out in the Introduction. With these techniques in hand, we have first extended more results of [24] by providing the polynomial bound $\gamma_{L}(G) \leq \gamma_{M}^{2}(G)$ for graphs $G$ without $C_{4}$ or $C_{6}$, and also by proving that, for arbitrary graphs $G$, any upper bound on $\gamma_{L}(G)$ in terms of $\gamma_{M}(G)$ is at least exponential. Subsequently, we have provided two bounds that surprisingly relate $\gamma_{M}(G)$ and $\psi(G)$, which have not been related so far: $\psi(G) \leq \gamma_{M}(G)$ whenever $\mathrm{g}(G) \geq 5$, and $\psi(G) \leq \gamma_{M}(G)+\gamma(G)$ for any graph $G$.

It would be interesting to characterize the trees $T$ with $\operatorname{dim}(T)=\gamma(T)$. Also, we could find new polynomial upper bounds on $\gamma_{L}(G)$ in terms of $\gamma_{M}(G)$ for other specific families of graphs. For arbitrary graphs, Theorem 3.2 could be improved by providing either a new construction (better than the graph $G_{s}$ ) or an upper bound on $\gamma_{L}(G)$ in terms of $\gamma_{M}(G)$. Concerning $\psi(G)$, besides settling Conjecture 4.8, it would be interesting to find similar bounds to those of Corollaries 4.5 and 4.7. Concretely, other classes of graphs $G$ with $\psi(G) \leq \gamma_{M}(G)$ could be found in order to extend Corollary 4.5. Also, as Corollary 4.7 is concerned with resolvability, location and domination, similar chains could be performed but using other related invariants such as the partition dimension, the total location-domination number, and the domatic number; among others. Furthermore, a different approach could produce new bounds involving parameters derived from other graph notions such as independence, symmetry, and connectivity. 


\section{References}

[1] R. F. Bailey and P. J. Cameron. Base size, metric dimension and other invariants of groups and graphs. Bull. Lond. Math. Soc., 43(2):209-242, 2011.

[2] C. Balbuena, F. Foucaud, and A. Hansberg. Locating-dominating sets and identifying codes in graphs of girth at least 5. Electron. J. Combin., 22(2):P2.15, 2015.

[3] M. Blidia, M. Chellali, F. Maffray, J. Moncel, and A. Semri. Locating-domination and identifying codes in trees. Australas. J. Combin., 39:219-232, 2007.

[4] D. L. Boutin. Identifying graph automorphisms using determining sets. Electron. J. Combin., 13(1):Research Paper 78, 12, 2006.

[5] J. Cáceres, C. Hernando, M. Mora, I. M. Pelayo, and M. L. Puertas. Locating-dominating codes: bounds and extremal cardinalities. Appl. Math. Comput., 220:38-45, 2013.

[6] J. Cáceres, C. Hernando, M. Mora, I. M. Pelayo, M. L. Puertas, C. Seara, and D. R. Wood. On the metric dimension of Cartesian products of graphs. SIAM J. Discrete Math., 21(2):423-441 (electronic), 2007.

[7] M. Čangalović, J. Kratica, V. Kovačević Vujčić, and M. Stojanović. Minimal doubly resolving sets of prism graphs. Optimization, 62(8):1037-1043, 2013.

[8] L.E. Celis, F. Pavetic, B. Spinelli, and P. Thiran. Budgeted sensor placement for source localization on trees. Electronic Notes in Discrete Mathematics, 50:65 - 70, 2015.

[9] G. Chartrand, L. Eroh, M. A. Johnson, and O. R. Oellermann. Resolvability in graphs and the metric dimension of a graph. Discrete Appl. Math., 105(1-3):99-113, 2000.

[10] X. Chen, X. Hu, and C. Wang. Approximation for the minimum cost doubly resolving set problem. Theoretical Computer Science, 609(3):526-243, 2016.

[11] X. Chen and C. Wang. Approximability of the minimum weighted doubly resolving set problem. In Computing and combinatorics, volume 8591 of Lecture Notes in Comput. Sci., pages 357-368. Springer, Cham, 2014.

[12] E. J. Cockayne and S. T. Hedetniemi. Towards a theory of domination in graphs. Networks, $7(3): 247-261,1977$.

[13] F. Foucaud. Decision and approximation complexity for identifying codes and locating-dominating sets in restricted graph classes. J. Discrete Algorithms, 31:48-68, 2015.

[14] F. Foucaud and M. A. Henning. Location-domination in line graphs. Discrete Math., 340(1):31403153, 2017.

[15] F. Foucaud, M. A. Henning, C. Löwenstein, and T. Sasse. Locating-dominating sets in twin-free graphs. Discrete Appl. Math., 200:52-58, 2016.

[16] F. Foucaud, G. B. Mertzios, R. Naserasr, A. Parreau, and P. Valicov. Identification, locationdomination and metric dimension on interval and permutation graphs. I. Bounds. Theoretical Computer Science, 668:43-58, 2017.

[17] F. Foucaud, G. B. Mertzios, R. Naserasr, A. Parreau, and P. Valicov. Identification, locationdomination and metric dimension on interval and permutation graphs. II. Algorithms and complexity. Algorithmica, 78(3):914-944, 2017.

[18] D. Garijo, A. González, and A. Márquez. The difference between the metric dimension and the determining number of a graph. Appl. Math. Comput., 249:487-501, 2014.

[19] D. Garijo, A. González, and A. Márquez. The resolving number of a graph. Discrete Math. Theor. Comput. Sci., 15(3):155-166, 2013.

[20] W. Goddard. Mastermind revisited. J. Combin. Math. Combin. Comput., 51:215-220, 2004.

[21] F. Harary and R. A. Melter. On the metric dimension of a graph. Ars Combinatoria, 2:191-195, 1976.

[22] T. W. Haynes, S. T. Hedetniemi, and P. J. Slater. Fundamentals of domination in graphs, volume 208 of Monographs and Textbooks in Pure and Applied Mathematics. Marcel Dekker Inc., New York, 1998.

[23] T. Haynes, D. Knisley, E. Seier, and Y. Zou. A quantitative analysis of secondary RNA structure using domination based parameters on trees. BMC Bioinformatics, 7(1):108, 2006.

[24] M. A. Henning and O. R. Oellermann. Metric-locating-dominating sets in graphs. Ars Combin., 73:129-141, 2004.

[25] C. Hernando, M. Mora, and I. M. Pelayo. Locating domination in bipartite graphs and their complements. ArXiv:1711.01951, 2017.

[26] C. Hernando, M. Mora, and I. M. Pelayo. Metric-locating-dominating partitions in graphs. ArXiv:1711.01086, 2017. 
[27] C. Hernando, M. Mora, and I. M. Pelayo. Nordhaus-Gaddum bounds for locating domination. European J. Combin., 36:1-6, 2014.

[28] A. Hertz. An ip-based swapping algorithm for the metric dimension and minimal doubly resolving set problems in hypercubes. Optimization Letters (2017), pages 1-13, 2017.

[29] I. Honkala and T. Laihonen. On locating-dominating sets in infinite grids. European J. Combin., $27(2): 218-227,2006$.

[30] J. Jiang, S. Wen, S. Yu, Y. Xiang, and W. Zhou. Identifying propagation sources in networks: State-of-the-art and comparative studies. IEEE Communications Surveys Tutorials, 19(1):465481, 2017.

[31] R. Kincaid, A. Oldham, and G. Yu. Optimal open-locating-dominating sets in infinite triangular grids. Discrete Appl. Math., 193:139-144, 2015.

[32] J. Kratica, M. Čangalović, and V. Kovačević-Vujčić. Computing minimal doubly resolving sets of graphs. Comput. Oper. Res., 36(7):2149-2159, 2009.

[33] J. Kratica, V. Kovačević-Vujčić, M. Cangalović, and M. Stojanović. Minimal doubly resolving sets and the strong metric dimension of Hamming graphs. Appl. Anal. Discrete Math., 6(1):63-71, 2012.

[34] J. Kratica, V. Kovačević-Vujčić, M. Čangalović, and M. Stojanović. Minimal doubly resolving sets and the strong metric dimension of some convex polytopes. Appl. Math. Comput., 218(19):97909801, 2012.

[35] J. McCoy and M. A. Henning. Locating and paired-dominating sets in graphs. Discrete Appl. Math., 157(15):3268-3280, 2009.

[36] N. Mladenović, J. Kratica, V. Kovačević-Vujčić, and M. Čangalović. Variable neighborhood search for metric dimension and minimal doubly resolving set problems. European J. Oper. Res., 220(2):328-337, 2012.

[37] O. Ore. Theory of graphs. American Mathematical Society Colloquium Publications, Vol. 38. American Mathematical Society, Providence, R.I., 1962.

[38] A. Sasireka and A.H. Nandhukishore. Applications of dominating set of graph in computer networks. International Journal of Engineering Sciences and Research Technology, 3(1):170-173, 2014.

[39] A. Sebö and E. Tannier. On metric generators of graphs. Math. Oper. Res., 29(2):383-393, 2004.

[40] J. L. Sewell and P. J. Slater. A sharp lower bound for locating-dominating sets in trees. Australas. J. Combin., 60:136-149, 2014.

[41] P. J. Slater. Dominating and reference sets in a graph. J. Math. Phys. Sci., 22(4):445-455, 1988.

[42] P. J. Slater. Fault-tolerant locating-dominating sets. Discrete Math., 249(1-3):179-189, 2002.

[43] P. J. Slater. Leaves of trees. In Proceedings of the Sixth Southeastern Conference on Combinatorics, Graph Theory, and Computing (Florida Atlantic Univ., Boca Raton, Fla., 1975), pages 549-559. Congressus Numerantium, No. XIV, Winnipeg, Man., 1975.

[44] S. Stephen, B. Rajan, C. Grigorious, and A. William. Resolving-power dominating sets. Appl. Math. Comput., 256:778-785, 2015.

[45] J. Suomela. Approximability of identifying codes and locating-dominating codes. Inform. Process. Lett., 103:28-33, 2007. 